\section{Cardiac Conditioning Ameliorates Cardiac Dysfunction Associated with Renal Hypertension in Rats}

Thomas F. Schaible, Gary J. Ciambrone, Joseph M. Capasso, and James Scheuer Division of Cardiology, Departments of Medicine and Physiology, Montefiore Medical Center and Albert Einstein College of Medicine, Bronx, New York 10467 bstract. To explore the effect of physiologic hypertrophy superimposed on pathologic hypertrophy, hearts from female control rats $(\mathrm{C})$, renal hypertensive rats $(\mathrm{H})$, rats conditioned with a 10-12 wk swimming program (Sw), and hypertensive rats trained by the swimming program (H-Sw) were perfused in an isolated working rat-heart apparatus. Systolic blood pressure was $\sim 100$ $\mathrm{mmHg}$ in $\mathrm{C}$ and $\mathrm{Sw}$ and was $160 \mathrm{mmHg}$ in $\mathrm{H}$ and $\mathrm{H}-$ Sw. The swimming program had no effect on blood pressure. Compared with $\mathrm{C}$, heart weight was increased by $30 \%$ in $\mathrm{Sw}, 47 \%$ in $\mathrm{H}$, and $77 \%$ in $\mathrm{H}-\mathrm{Sw}$. At high preload and afterload, cardiac output (milliliters per gram dry LV weight) was decreased in $\mathrm{H}$, increased in $\mathrm{Sw}$, and partially restored towards normal in $\mathrm{H}-\mathrm{Sw}$. Ejection fraction, percent fractional shortening, and mean velocity of circumferential fiber shortening were enhanced in Sw, depressed in $\mathrm{H}$, and normalized in $\mathrm{H}-\mathrm{Sw}$ when compared with C. Coronary flow and myocardial oxygen consumption in this series of hearts were depressed in $\mathbf{H}$, with no restoration in $\mathrm{H}-\mathrm{Sw}$, but coronary effluent lactate/ pyruvate ratios were only elevated in the hearts of $\mathrm{H}-\mathrm{Sw}$. Coronary vascular responses were examined in a second series of experiments which used microspheres. In this series, the depressed coronary flow observed in $\mathbf{H}$ was partially restored towards normal in $\mathrm{H}-\mathrm{Sw}$ and the inner/ outer myocardial flow ratio was normal when hearts were

\footnotetext{
This work was presented in part at the Annual Scientific Session of the American College of Cardiology, New Orleans, LA, March, 1983.

Dr. Schaible is the recipient of a Young Investigator Research Award (HL-25372).

Received for publication 14 February 1983 and in revised form 3 January 1984.
}

J. Clin. Invest.

(c) The American Society for Clinical Investigation, Inc.

0021-9738/84/04/1086/09 \$1.00

Volume 73, April 1984, 1086-1094 perfused at $140 \mathrm{~cm}$ aortic pressure but was somewhat depressed in both $\mathrm{H}$ and $\mathrm{H}-\mathrm{Sw}$ when the hearts were perfused at $80 \mathrm{~cm}$ aortic pressure.

These studies demonstrate that hypertrophic hearts from renal hypertensive rats have diminished coronary flow and depressed cardiac function when they are studied in the isolated working heart apparatus, yet there is no evidence of myocardial ischemia. Superimposition of a chronic swimming program results in increased hypertrophy but restoration of cardiac function partially or completely to normal. Thus, pathologic and physiologic hypertrophy are biologically distinct entities. Physiologic hypertrophy may partially ameliorate the defects associated with pathologic hypertrophy.

\section{Introduction}

Cardiac hypertrophy due to systolic overload of the ventricles may be associated with normal or depressed cardiac function (1-3). However, in rats this is generally associated with depressed myocardial contractility and ventricular function (4-7). On the other hand, hypertrophy associated with hyperthyroidism or with physical training has frequently been found to be associated with enhanced myocardial contractile performance and ventricular function (8). It was our assumption that when cardiac hypertrophy due to systolic overload was associated with depressed function this might be worsened by adding a physiologic load that exaggerates the hypertrophy.

Contractile protein abnormalities usually parallel the physiologic alterations mentioned above; that is, a depression in myosin-associated ATPase activity with pathologic hypertrophy and enhanced activity with physiologic hypertrophy or hyperthyroidism (9). We recently reported that hypertensive rats with cardiac hypertrophy have depressed actin activated myosin ATPase activity, but that this contractile protein alteration is reversed when similar hypertensive animals are trained by a chronic swimming program, despite the fact that the cardiac hypertrophy becomes exaggerated (10). Parallel alterations were observed in myosin isoenzyme patterns in that the pathologic hypertrophy alone resulted in a shift from a predominance of 
the high ATPase isoenzyme $\left(\mathrm{V}_{1}\right)$ of rat cardiac myosin to a predominant pattern of a lower ATPase activity cardiac myosin isoenzyme $\left(V_{3}\right)$, and that the superimposition of the swimming load reversed this abnormal pattern.

The above findings with contractile protein shifts suggested the possibility that a chronic swimming program might improve myocardial contractile performance and cardiac function in hypertensive rats even though the cardiac hypertrophy would be exaggerated. The present experiments were conducted to explore that possibility.

\section{Methods}

Animal models. Female Wistar rats, aged 10 wk and weighing $\sim 175 \mathrm{~g}$ at the beginning of the experiment, were made hypertensive by placing silver clips $(0.18-0.25 \mathrm{~mm}$ i.d.) around the left renal artery as described previously $(4,7)$. Sham animals (controls) were also prepared from the same shipment as the experimental animals and had the same surgical procedure except for the placement of the renal artery clips. 3 wk after surgery, blood pressure was measured by the tail-cuff method under light ether anesthesia (7). When the blood pressure exceeded $150 \mathrm{mmHg}$ the animals were considered to be hypertensive. Half of the animals in the control and hypertensive groups were subjected to a swimming program and the other half remained cage-confined (sedentary). The exercise program, described previously (11-13), consisted of swimming $5 \mathrm{~d} / \mathrm{wk}, 75 \mathrm{~min}$ twice a day for $10 \mathrm{wk}$. At the completion of this period, exercised and sedentary animals were anesthetized with ether, their hearts were removed and studied in the isolated working rat heart apparatus.

Heart perfusions. In the first series of experiments hearts were perfused for analysis of pump function, myocardial function, and cardiac metabolism. Full descriptions of the isolated working rat heart apparatus have been published previously (11-13). The perfusate was a modified Krebs-Henseleit buffer at $37^{\circ} \mathrm{C}$ gassed with a $95 \% \mathrm{O}_{2}-5 \% \mathrm{CO}_{2}$ mixture and containing $15 \mathrm{mM}$ glucose, $0.01 \mathrm{U} / \mathrm{ml}$ regular insulin (Eli Lilly International Corp., Indianapolis, IN), and $2.0 \mathrm{mM}$ calcium with 0.5 $\mathrm{mM}$ EDTA, yielding $1.5 \mathrm{mM}$ free calcium. The perfusate was not recirculated. Left ventricular (LV)' ${ }^{1}$ pressure was measured through a 2.5 $\mathrm{cm}$ polyethylene (PE-60) catheter inserted through the apex of the heart and attached to a Statham P23dB strain gauge pressure transducer. A second catheter (PE-20) was placed through the LV apex for dye injection. Aortic pressure was measured from a sidearm on the aortic cannula $\sim 7-8 \mathrm{~mm}$ above the aortic valve. The frequency responses of the pressure measuring systems were flat $\pm 10 \%$ to $30 \mathrm{~Hz}$. Instantaneous aortic flow was measured from a cannulating $2.5 \mathrm{~mm}$ i.d. flow probe (StathamGould, Oxnard, CA; frequency response set to $50 \mathrm{~Hz}$ ) inserted in the aortic outflow tubing. Coronary flow was measured directly as right heart outflow and cardiac output was measured as aortic flow plus coronary flow. Dye concentrations in the aorta were measured by a densitometer system placed into the aortic cannula. All hearts were paced from the right atrium at a rate of 340 beats $/ \mathrm{min}$. Oxygen tension was measured in the perfusate from the left atrial reservoir and from a pulmonary arterial catheter. Arteriovenous differences in oxygen tension were converted to oxygen consumption (milliliters $\mathrm{O}_{2}$ per gram per min) by multiplying the arteriovenous oxygen tension by coronary flow and

1. Abbreviations used in this paper: ADP, aortic diastolic pressure; EDP, end-diastolic pressure; $C$, controls; $\mathrm{H}$, hypertensives; $\mathrm{H}-\mathrm{Sw}$, hypertensive swimmers; L/P, lactate/pyruvate; LAP, left atrial pressure, LV, left ventricle; Sw, swimmers; Vcf, velocity of circumferential fiber shortening. the appropriate Bunsen coefficient and dividing by LV dry weight Whenever possible, one heart from each group was perfused on a given day.

Cardiac function was assessed at two levels of preload at each of three levels of afterload. Afterload was varied by changing the height of the aortic overflow chamber. This procedure determines the aortic diastolic pressure (ADP). In the present study, the aortic overflow chamber was set up to be 80,110 , and $140 \mathrm{~cm}$ above the heart, which resulted in aortic diastolic pressures of 59,81 , and $103 \mathrm{mmHg}$, respectively.

Preload at each level of ADP was varied by changing the height of the left atrial reservoir to produce varying levels of left atrial pressure (LAP). The hearts in the present study varied widely in size, and since the left atrial catheter was of the same diameter for all hearts, equilibration between the LAP and the ventricular diastolic pressure was not always achieved in larger hearts. Therefore, we attempted to equate the preload in all hearts by using end-diastolic pressure (EDP) as the end-point rather than the LAP. The EDP could be seen readily on the LV pressure trace under high amplification and could be set to a calibrated value by changing the LAP. Ventricular function was examined at two levels of preload, which will be called moderate and high. The moderate preload corresponded to an EDP of 5-6 $\mathrm{mmHg}$, while the high preload corresponded to an EDP of 9-14 $\mathrm{mmHg}$. We observed that variations in EDP within the range defined as the high preload condition did not markedly alter cardiac output or left ventricular pressure. Thus, the values for pump performance obtained at the high LAP represent the maximal Frank-Starling response for any heart at a given ADP and at the paced heart rate of 340 beats $/ \mathrm{min}$. In addition, since preliminary studies indicated that a higher EDP was necessary in hearts of hypertensive animals to produce a similar estimated end-diastolic wall stress as in hearts from control animals, the EDP was set $\sim 1-2 \mathrm{mmHg}$ higher in hearts from hypertensive animals.

After a 10-15 min period of retrograde perfusion, during which catheters for LV pressure and dye injection were positioned, antegrade perfusion was begun by unclamping the left atrial catheter. After a 10min equilibration period, in which the pressure transducers and flow probe were zeroed, measurements of dynamics were made at the moderate and high LAP at an aortic height of $80 \mathrm{~cm} \mathrm{H}_{2} \mathrm{O}$, followed by measurements at $110 \mathrm{~cm} \mathrm{H}_{2} \mathrm{O}$ and finally at $140 \mathrm{~cm} \mathrm{H}_{2} \mathrm{O}$. At each aortic height, measurements were first determined at the moderate LAP and then at the high LAP. After $3 \mathrm{~min}$ at each loading condition, records were made of dynamics, multiple dye dilution curves were recorded, and coronary flow and cardiac output were measured over a 1-min period. All analogue data (dye concentration, aortic flow, and LV and aortic pressure) were stored on magnetic tape for later analysis on a digital computer.

Ejection fraction was determined by a dye-dilution method. A full description of this method and the validation for estimating ventricular volumes has been published previously (13). For the 35 hearts that were perfused in the current study, ejection fraction at any given loading condition was the mean of $\sim 18$ values with a coefficient of variation of $\sim 7 \%$. End-diastolic volume was calculated by dividing the directly measured stroke volume (cardiac output divided by the heart rate) by the ejection fraction.

Flow and pressure analogue data were digitized at 660 samples/s on a Mod Comp Max II/2 digital computer and the measurements of cardiac dynamics were determined as previously described (11-13). Instantaneous wall stress was estimated from the LaPlace equation and expressed in $\mathrm{g} / \mathrm{cm}^{2}$. Mean values for velocity of circumferential fiber shortening (Vcf) and wall stress during ejection were obtained by integration (12). End-diastole (mitral valve closure) was defined as the last $L V$ pressure for which $\mathrm{d} P / \mathrm{d} t$ was $<500 \mathrm{mmHg} / \mathrm{s}(13)$. 
Lactate and pyruvate concentrations were measured in samples of the coronary effluent to estimate the myocardial production of these metabolites. Since the coronary effluent was not recirculated and neither lactate nor pyruvate was added to the perfusion buffer, arterial concentrations were assumed to be zero. Lactate and pyruvate concentrations were determined by the enzymatic methods of Hohorst (14) and Segal et al. (15), respectively.

At the end of each experiment the atria and great vessels were dissected free and the right ventricular free wall was removed. The LV (including the septum) was weighed to determine wet weight. A small piece of the LV $(\sim 0.2 \mathrm{~g}$ wet $\mathrm{wt})$ was dried to constant weight in an oven to calculate LV dry weight.

Microsphere studies. To explore the details of coronary flow distribution, a second series of hearts was perfused using radiolabeled microspheres. The experimental preparation was similar to that described above except that a sidearm was placed in the tubing between the left atrial reservoir and the left atrium for injection of the microspheres.

Determinations of flow distribution were made at the high preload condition at two aortic chamber heights, 80 and $140 \mathrm{~cm} \mathrm{H}_{2} \mathrm{O} .15-\mu \mathrm{m}$ diam microspheres labeled with $\mathrm{Sc}^{46}$ or $\mathrm{Sn}^{113}$ were resuspended in $6 \%$ dextran, to which a drop of Tween 80 was added, and ultrasonicated for $10 \mathrm{~min}$ before injection. A $0.2-\mathrm{ml}$ volume of this suspension (calculated to deliver 100,000 microspheres) was injected into the left atrial line and flushed with $2.5 \mathrm{ml}$ of perfusion buffer. Measurements of coronary flow and dynamics were made before, during, and after the microsphere injection to ensure that the preparation remained stable, and that the directly measured total coronary flow was constant. These measurements varied $<5 \%$ in individual hearts.

At the completion of the study, the left and right atrial appendages and great vessels were removed and discarded. The right ventricular free wall and apical tip were cut away and weighed. The left ventricle (including the septum) was breadloafed into basal and apical rings and each ring was separated into a septal and free wall half. Each of these four pieces was then divided into inner and outer sections and each of the resulting eight pieces was weighed. The inner/outer weight ratio was $\sim 0.6$ and was not different amongst the four experimental groups.

Tissue samples were counted in a Searle gamma counter and the net counts (counts per minute) for each piece were corrected for background and energy overlap. Flow to each piece was calculated by the following equation: Flow $_{\text {piece }}(\mathrm{ml} / \mathrm{g}$ per $\mathrm{min})=(\mathrm{cpm}$ piece $) /(\mathrm{cpm}$ heart $)$ $X$ total flow $(\mathrm{ml} / \mathrm{min}) \div$ piece wt $(\mathrm{g})$. Total flow was that coronary flow measured directly during the $1 \mathrm{~min}$ that included the microsphere injection. cpm heart represents the sum of the counts from all pieces. The values for inner/outer flow ratio for each heart were averaged from the values obtained for the four left ventricular pieces.

Statistical analysis. Results were submitted to a two-factor analysis of variance utilizing conditioning status (sedentary/swimmer) or blood pressure status (normotensive/hypertensive) as the two factors. The mean square error for within groups from this analysis was then used in a Newman-Keuls multiple comparison test (16) to test for differences between hearts from any two groups. Probabilities are reported at the 0.05 and 0.001 level.

\section{Results}

Blood pressure and body and heart weights. Blood pressure measured at the completion of the 10-wk swimming program was $101 \pm 7 \mathrm{mmHg}$ (mean \pm SE) in controls (C), 98 \pm 4 in swimmers $(\mathrm{Sw}), 157 \pm 8$ in hypertensives $(\mathrm{H})$ and $159 \pm 6$ in hypertensive swimmers (H-Sw). Although differences between any hypertensive and normotensive group were highly significant $(P<0.001)$, there were no significant differences within normotensive or hypertensive groups. Thus, as we reported previously, chronic swimming did not alter the level of blood pressure in normotensive or hypertensive animals (10).

Table I shows the heart and body weight relationships for the experimental groups at the time of death. Body weights were similar among all four groups. Compared with $\mathrm{C}$, the dry heart weight was $30 \%$ greater in $\mathrm{Sw}, 46 \%$ greater in $\mathrm{H}$, and $76 \%$ greater in $\mathrm{H}-\mathrm{Sw}$ (all differences $P<0.001$ ). Hearts from $\mathrm{H}-\mathrm{Sw}$ were significantly more hypertrophied than hearts from $\mathrm{H}(P<0.001)$. A similar relationship was observed for the left ventricular weights. Right ventricular weights were greater in $\mathrm{Sw}, \mathrm{H}$, and $\mathrm{H}-\mathrm{Sw}$ than in $\mathrm{C}$, but was less in $\mathrm{H}$ than in Sw $(P<0.05)$ and the same in Sw and H-Sw.

Heart perfusions. Table II and Fig. 1-3 show results from the isolated perfused heart studies. Table II shows results obtained at high preload at the three levels of aortic chamber height (ADP), for measurements of coronary flow, myocardial oxygen utilization and metabolism, and cardiac function. Coronary flow per gram LV was significantly lower in both hypertensive groups compared with either normotensive group at all loading conditions. The values for coronary flow tended to be higher in $\mathrm{H}-$

Table I. Heart and Body Weights

\begin{tabular}{|c|c|c|c|c|c|c|}
\hline & BW & DHW & DLVW & DRVW & $\mathrm{D} / \mathrm{W}$ & DLVW/DHW \\
\hline & $g$ & $m g$ & $m g$ & $m g$ & & \\
\hline Sw (7) & $278 \pm 9$ & $184 \pm 6^{*}$ & $149 \pm 6^{*}$ & $35 \pm 1^{*}$ & $0.208 \pm 0.005$ & $0.807 \pm 0.007 \ddagger$ \\
\hline$H(10)$ & $276 \pm 6$ & $206 \pm 6 \S$ & $178 \pm 5 \S$ & $29 \pm 1^{11}$ & $0.206 \pm 0.003$ & $0.861 \pm 0.003 \S$ \\
\hline H-Sw (9) & $272 \pm 3$ & $249 \pm 7 * \S \pi$ & $212 \pm 6 * \S \pi$ & $37 \pm 1 * \S$ & $0.210 \pm 0.003$ & $0.852 \pm 0.005 \S \pi$ \\
\hline
\end{tabular}

Results are the mean \pm SEM. The number of hearts are shown in parentheses. C, control; Sw, swimmer; H, hypertensive; BW, body weight; DHW, dry heart weight; DLVW, dry left ventricular weight; DRVW, dry right ventricular weight; $D / W$, dry to wet heart weight ratio. ${ }^{*} P$ $<0.001$; Sw vs. C or H-Sw vs. H. $\ddagger P<0.05$, Sw vs. C or H-Sw vs. H. $\$ P<0.001 ; \mathrm{H}$ vs. C or H-Sw vs. C. $\| P<0.05, \mathrm{H}$ vs. C or H-Sw vs. C. I $P<0.001, \mathrm{H}-\mathrm{Sw}$ vs. Sw. 
Table II. Measurements of Coronary Flow, Myocardial Oxygen Utilization and Metabolism, and Cardiac Function at High Left Atrial Pressure and Varying Aortic Heights

\begin{tabular}{|c|c|c|c|c|c|}
\hline & Aortic height & C (9) & Sw (7) & $H(10)$ & H-Sw (9) \\
\hline & $\mathrm{cm}$ & & & & \\
\hline \multirow{3}{*}{$\begin{array}{l}\text { Coronary flow } \\
\qquad\left(\mathrm{ml} / \mathrm{g}^{-1} \text { per } \mathrm{min}\right)\end{array}$} & 80 & $104 \pm 2$ & $109 \pm 2$ & $64 \pm 4 \S$ & $66 \pm 4 \S \pi$ \\
\hline & 110 & $132 \pm 2$ & $136 \pm 3$ & $84 \pm 5 \S$ & $90 \pm 4 \S \pi$ \\
\hline & 140 & $155 \pm 5$ & $155 \pm 4$ & $101 \pm 5 \S$ & $108 \pm 5 \S \rrbracket$ \\
\hline \multirow{3}{*}{$\begin{array}{l}\mathrm{O}_{2} \text { extraction } \\
(\mathrm{ml} / \mathrm{ml})\end{array}$} & 80 & $0.72 \pm 0.01$ & $0.76 \pm 0.01 \ddagger$ & $0.83 \pm 0.01 \S$ & $0.84 \pm 0.01 \S \pi$ \\
\hline & 110 & $0.69 \pm 0.01$ & $0.73 \pm 0.01 \ddagger$ & $0.80 \pm 0.01 \S$ & $0.80 \pm 0.01 \S \pi$ \\
\hline & 140 & $0.66 \pm 0.02$ & $0.69 \pm 0.02$ & $0.77 \pm 0.02 \S$ & $0.78 \pm 0.01 \S^{* *}$ \\
\hline \multirow{3}{*}{$\begin{array}{l}\text { Lactate production } \\
\qquad(\mu \mathrm{mol} / \mathrm{g} \text { per } \mathrm{min})\end{array}$} & 80 & $8.2 \pm 0.7$ & $11.3 \pm 1.2$ & $17.4 \pm 1.8 \S$ & $21.1 \pm 1.3 \S \pi$ \\
\hline & 110 & $8.6 \pm 0.8$ & $11.1 \pm 0.7$ & $13.8 \pm 1.1$ & $17.0 \pm 2.2^{\| * *}$ \\
\hline & 140 & $9.1 \pm 0.8$ & $10.5 \pm 1.2$ & $12.3 \pm 1.0$ & $14.5 \pm 1.5^{\prime \prime}$ \\
\hline \multirow[t]{3}{*}{$\mathrm{L} / \mathrm{P}$} & 80 & $5.6 \pm 0.3$ & $6.6 \pm 0.6$ & $11.7 \pm 1.1 \S$ & $15.4 \pm 0.7 \ddagger \S \pi$ \\
\hline & 110 & $5.8 \pm 0.4$ & $6.7 \pm 0.5$ & $9.4 \pm 0.9^{\prime \prime}$ & $11.6 \pm 1.2 \S^{* *}$ \\
\hline & 140 & $6.7 \pm 0.7$ & $6.8 \pm 0.5$ & $9.4 \pm 1.0$ & $11.5 \pm 1.0^{11 * *}$ \\
\hline \multirow{3}{*}{$\begin{array}{l}\mathrm{MVO}_{2} \\
\quad\left(\mathrm{ml} \mathrm{O}_{2} / g \text { per min }\right)\end{array}$} & 80 & $1.55 \pm 0.03$ & $1.73 \pm 0.02 \ddagger$ & $1.10 \pm 0.07 \S$ & $1.15 \pm 0.06 \S \pi$ \\
\hline & 110 & $1.88 \pm 0.05$ & $2.05 \pm 0.04$ & $1.40 \pm 0.07 \S$ & $1.51 \pm 0.07 \S \pi$ \\
\hline & 140 & $2.10 \pm 0.09$ & $2.21 \pm 0.04$ & $1.63 \pm 0.07 \S$ & $1.75 \pm 0.0811 \pi$ \\
\hline \multirow{3}{*}{$\begin{array}{l}\text { Stroke work } \\
\quad\left(\text { ergs } \cdot 10^{5} \cdot g^{-1}\right)\end{array}$} & 80 & $1.90 \pm 0.06$ & $2.25 \pm 0.06 \ddagger$ & $1.26 \pm 0.08 \S$ & $1.37 \pm 0.05 \S \pi$ \\
\hline & 110 & $1.99 \pm 0.06$ & $2.35 \pm 0.07^{*}$ & $1.45 \pm 0.07 \S$ & $1.68 \pm 0.05 \ddagger^{\| 1 / \pi}$ \\
\hline & 140 & $1.93 \pm 0.08$ & $2.32 \pm 0.09 \ddagger$ & $1.46 \pm 0.07 \S$ & $1.78 \pm 0.05 \ddagger \pi$ \\
\hline \multirow[t]{3}{*}{ Efficiency } & 80 & $0.195 \pm 0.007$ & $0.205 \pm 0.005$ & $0.181 \pm 0.005$ & $0.191 \pm 0.005$ \\
\hline & 110 & $0.168 \pm 0.006$ & $0.182 \pm 0.005$ & $0.166 \pm 0.004$ & $0.178 \pm 0.005$ \\
\hline & 140 & $0.148 \pm 0.008$ & $0.166 \pm 0.006$ & $0.143 \pm 0.005$ & $0.163 \pm 0.005$ \\
\hline \multirow{3}{*}{$\begin{array}{l}\text { Fractional shortening } \\
\text { at midwall }(\%)\end{array}$} & 80 & $19.1 \pm 0.5$ & $20.3 \pm 0.7$ & $15.2 \pm 0.5 \S$ & $16.8 \pm 0.4 \ddagger^{11 \pi}$ \\
\hline & 110 & $16.8 \pm 0.4$ & $18.6 \pm 0.5 \ddagger$ & $14.5 \pm 0.4 \S$ & $16.9 \pm 0.3^{*, * *}$ \\
\hline & 140 & $13.7 \pm 0.4$ & $16.0 \pm 0.6 \ddagger$ & $12.3 \pm 0.5^{\prime \prime}$ & $15.1 \pm 0.3^{* \|}$ \\
\hline
\end{tabular}

Results are the mean $\pm \mathrm{SE}$ for the number of hearts shown in parentheses. $\mathrm{MVO}_{2}$, myocardial oxygen consumption. Statistical comparisons were made at each aortic height and the notations are the same as in Table I. ${ }^{* *} P<0.05, \mathrm{H}-\mathrm{Sw}$ vs. Sw.

Sw compared with $\mathrm{H}$ although they did not achieve statistical significance. Associated with the lower coronary flow values in the hypertensive groups were significantly greater values for oxygen extraction. However, as coronary flow increased with increases in ADP, oxygen extraction decreased in all groups $(P$ $<0.01$.

Myocardial oxygen consumption was significantly less in both hypertensive groups compared with either normotensive group. It tended to be greater in hearts from swimmers compared with hearts from sedentary animals within normotensive and hypertensive groups, but only achieved statistical significance in Sw vs. $C$ at $80 \mathrm{~cm}$ ADP. The effect of increasing ADP on lactate production and the lactate/pyruvate $(\mathrm{L} / \mathrm{P})$ ratio was quite different in the hearts from normotensive and hypertensive animals. Since lactate and pyruvate are not included in the perfusion buffer the amounts of these metabolites in the coronary effluent reflect myocardial production. Lactate production and the $\mathrm{L} / \mathrm{P}$ ratio remained essentially the same in both normotensive groups regardless of the ADP. However, there were declines ( $P$ $<0.01$ ) in these values in both hypertensive groups as the ADP was increased. At an ADP of $80 \mathrm{~cm} \mathrm{H}_{2} \mathrm{O}$, values for lactate production and $\mathrm{L} / \mathrm{P}$ ratio were significantly greater in $\mathrm{H}$ and $\mathrm{H}-\mathrm{Sw}$ than in $\mathrm{C}$, but at an ADP of $140 \mathrm{~cm} \mathrm{H}_{2} \mathrm{O}$, they were not different between $\mathrm{H}$ and $\mathrm{C}$, but were significantly elevated in $\mathrm{H}$-Sw compared with $\mathrm{C}$.

Since differences between hearts from swimmers and sedentary animals were most obvious at $140 \mathrm{~cm} \mathrm{H}_{2} \mathrm{O}$ ADP, results obtained at the moderate and high preloads at this aortic pressure are shown in Figs. 1-3.

Fig. 1 shows values for end-diastolic volume and pressure and for end-diastolic wall stress. Absolute end-diastolic volumes were significantly greater in $\mathrm{Sw}$ and $\mathrm{H}-\mathrm{Sw}$ than in $\mathrm{C}$ at both 

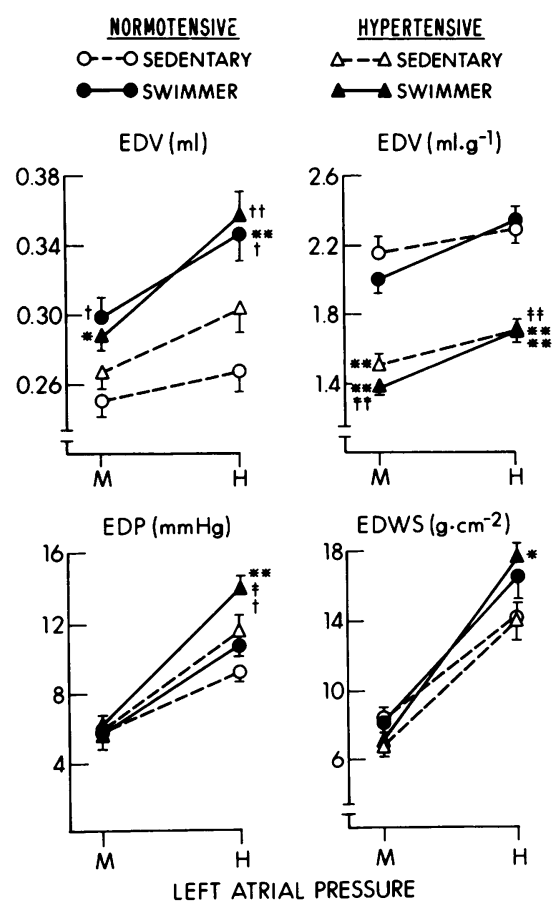

Figure 1. End diastolic volume (EDV) (milliliters and milliliters per gram), EDP, and end diastolic wall stress (EDWS) in perfused hearts. Values shown are for perfusions with aortic diastolic pressure at 140 $\mathrm{cm} \mathrm{H}_{2} \mathrm{O}$. M, moderate and $\mathrm{H}$, high preload (defined in text). Results are mean \pm SE for 9 sedentary, 7 swimmers, 10 hypertensive sedentary, and 9 hypertensive swimmers. The symbols for $P$ values are: $\dagger$ $<0.05$ and $+\dagger<0.001$ in Sw vs. $\mathrm{C}$ or $\mathrm{H}-\mathrm{Sw}$ vs. $\mathrm{H} ;{ }^{*}<0.05$ and ${ }^{* *}$ $<0.001$ in $\mathrm{H}$ vs. $\mathrm{C}$ or $\mathrm{H}$-Sw vs. $\mathrm{C} ; \ddagger<0.05$ and $\ddagger \ddagger<0.001$ in $\mathrm{H}-\mathrm{Sw}$ vs. Sw. $O$, normotensive sedentary; $\bullet$, normotensive swimmer; $\Delta$, hypertensive sedentary; $\Delta$, hypertensive swimmer.

moderate (M) and high (H) LAP. At the high LAP, values were also greater in $\mathrm{H}$-Sw than in $\mathrm{H}$. Values were not different between $\mathrm{H}$ and $\mathrm{C}$ at either preload. When volume was normalized for dry LV weight, there were no differences between hearts from swimmers or sedentary animals within the normotensive or hypertensive groups. However, both $\mathrm{H}$ and $\mathrm{H}-\mathrm{Sw}$ had significantly lower values than $\mathrm{C}$ at both preloads. No differences were observed for end-diastolic pressure or wall stress between the groups at the moderate LAP, but end-diastolic pressures were greater in $\mathrm{H}-\mathrm{Sw}$ compared with $\mathrm{C}$, Sw, or $\mathrm{H}$ at the high LAP. End-diastolic wall stress was slightly but significantly increased only in $\mathrm{H}-\mathrm{Sw}$ as compared with $\mathrm{C}$ at the high preload.

Fig. 2 shows measurements of pump performance in the four groups. Values for cardiac output are shown expressed as milliliters per minute and normalized per gram dry LV weight. When expressed as milliliters per minute, cardiac output was similar in Sw and H-Sw and in $\mathrm{C}$ and $\mathrm{H}$. Values in Sw and $\mathrm{H}-$ $\mathrm{Sw}$ were greater at both preloads than in $\mathrm{C}$ or $\mathrm{H}$. When expressed per gram dry LV, cardiac output was significantly lower in the

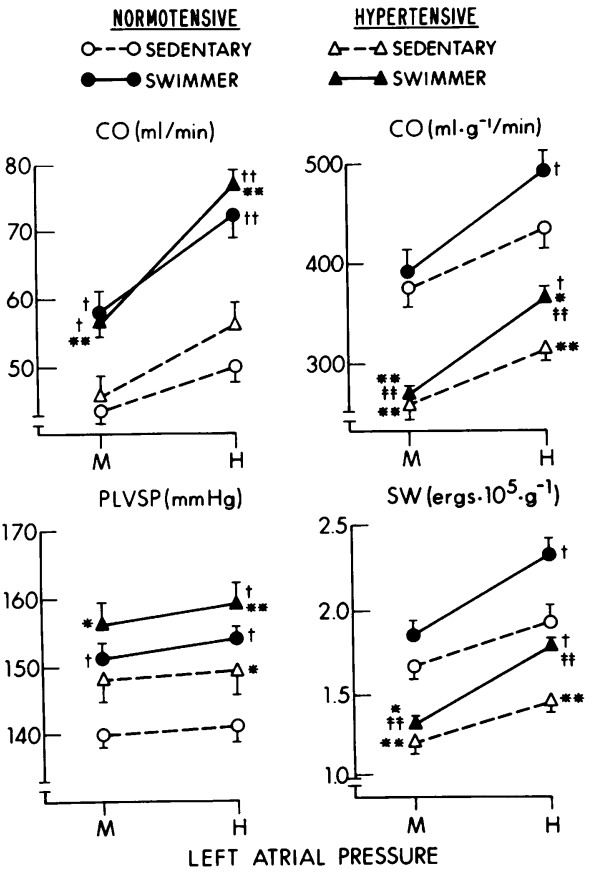

Figure 2. Pump function in perfused hearts. $\mathrm{CO}$, cardiac output; PLVSP, peak left ventricular systolic pressure; SW, stroke work. All other symbols and abbreviations are the same as in Fig. 1.

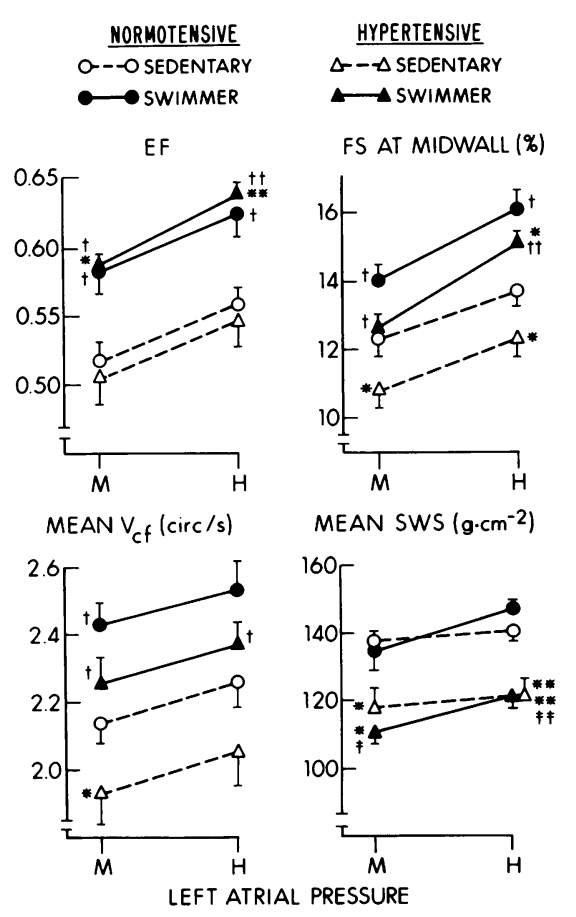

Figure 3. Myocardial function in perfused hearts. EF, ejection fraction; FS, fractional shortening; SWS, mean systolic wall stress. Symbols and abbreviations are the same as in Fig. 1. 
hypertensive groups than in the normotensive groups at all loading conditions. However, cardiac output per gram was greater in $\mathrm{Sw}$ as compared with $\mathrm{C}$ and in $\mathrm{H}-\mathrm{Sw}$ compared with $\mathrm{H}$ at the high LAP, a result that is consistent with our previous observations that hearts of trained animals must be stressed in order to uncover the effects of training. Within normotensive or hypertensive groups, peak left ventricular systolic pressure was greater in hearts from swimmers than in hearts from sedentary animals at both preloads. At the high LAP, peak pressure was greater in $\mathbf{H}$ than in $\mathrm{C}$. Values for stroke work under all loading conditions are shown in Table II and at high aortic pressure in Fig. 2. Values for stroke work (per gram LV) were significantly greater in Sw as compared with $C$ at all ADP and in $\mathrm{H}-\mathrm{Sw}$ compared with $\mathrm{H}$ at higher levels of ADP. Values for $\mathrm{H}$ were lower than those in $\mathrm{C}$ at all ADP but were lower in $\mathrm{H}$ Sw only at the lower levels of ADP. At $140 \mathrm{~cm} \mathrm{H}_{2} \mathrm{O}$ ADP, stroke work was markedly depressed in $\mathrm{H}$ vs. $\mathrm{C}$, but was similar to $\mathrm{C}$ in $\mathrm{H}-\mathrm{Sw}$. Differential responses for stroke work to increasing ADP also occurred among the groups. As ADP was increased from 80 to $140 \mathrm{~cm} \mathrm{H}_{2} \mathrm{O}, 2,3,16$, and $30 \%$ increases in stroke work were observed in $\mathrm{C}, \mathrm{Sw}, \mathrm{H}$, and $\mathrm{H}-\mathrm{Sw}$, respectively.

Values for external efficiency are also shown in Table II. These were not statistically different for any comparison between groups although a trend for higher values was observed in hearts from Sw and H-Sw. Also, external efficiency declined $(P<0.001)$ as aortic pressure was raised.

Fig. 3 shows results for ejection fraction, fractional shortening, velocity of shortening, and force development. Ejection fraction was similar in Sw and $\mathrm{H}-\mathrm{Sw}$, and greater in both of these groups than in either $\mathrm{C}$ or $\mathrm{H}$. Compared with $\mathrm{C}$, percent midwall shortening was significantly depressed in $\mathrm{H}$, but was significantly greater in Sw at both moderate and high preloads. Shortening was the same as $\mathrm{C}$ in $\mathrm{H}-\mathrm{Sw}$ at moderate preload and greater in $\mathrm{H}-\mathrm{Sw}$ than in both $\mathrm{C}$ and $\mathrm{H}$ at the high preload. Table II details percent midwall shortening under all loading conditions. Midwall fractional shortening was greater in Sw than $\mathrm{C}$ and in $\mathrm{H}-\mathrm{Sw}$ than $\mathrm{H}$ particularly at the high ADP. Shortening was less in $\mathrm{H}$ than in $\mathrm{C}$ at all ADP. However, although shortening was less in $\mathrm{H}-\mathrm{Sw}$ than $\mathrm{C}$ at $80 \mathrm{~cm} \mathrm{H}_{2} \mathrm{O}$ ADP, it was greater in $\mathrm{H}-\mathrm{Sw}$ at $140 \mathrm{~cm} \mathrm{H}_{2} \mathrm{O}$.

$\mathrm{Vcf}$ was significantly greater in $\mathrm{Sw}$ than in $\mathrm{C}$ at the moderate preload and greater in $\mathrm{H}-\mathrm{Sw}$ than in $\mathrm{H}$ at both the moderate and high preload. Differences between $\mathrm{Sw}$ and $\mathrm{C}$ at the high LAP were of borderline significance $(P<0.06)$. Velocity was lower in $\mathrm{H}$ compared with $\mathrm{C}$ at the moderate preload and marginally lower $(P<0.06)$ at the high preload. Mean systolic wall stress was similar between hearts from swimmers and sedentary animals within the normotensive or hypertensive groups. Values in both hypertensive groups were lower than in either normotensive group.

Microsphere studies. Results for the second series of experiments in which myocardial flow distribution was studied are shown in Table III. Similar to the perfusion studies described above, the total coronary flow per gram wet $\mathrm{LV}$ was less in both hypertensive groups than in either normotensive group. However, in this series of hearts, a greater coronary flow was observed in $\mathrm{H}-\mathrm{Sw}$ compared with $\mathrm{H}$ at both 80 and $140 \mathrm{~cm} \mathrm{H}_{2} \mathrm{O}$ ADP and in Sw compared with $\mathrm{C}$ at $140 \mathrm{~cm} \mathrm{H}_{2} \mathrm{O}$ ADP. Similar differences between hearts from swimmers and sedentary animals were observed for left ventricular myocardial flow. Conversely, right ventricular myocardial flow was not different between any of the groups of hearts at any aortic height. At $80 \mathrm{~cm} \mathrm{H}_{2} \mathrm{O}$ ADP the inner/outer flow ratio was significantly lower in both $\mathrm{H}$ and $\mathrm{H}-\mathrm{Sw}$ compared with $\mathrm{C}$. However, at $140 \mathrm{~cm} \mathrm{H}_{2} \mathrm{O}$ ADP, there were no differences in any group comparison for this measure of transmural flow distribution.

\section{Discussion}

The present study investigated the effects of superimposing an exercise program upon cardiac function and coronary flow in hearts of rats with renal hypertension. This report confirms that

Table III. Myocardial Flow Distribution

\begin{tabular}{|c|c|c|c|c|c|}
\hline & Aortic height & C (5) & Sw (5) & H (5) & $\mathrm{H}-\mathrm{Sw}(4)$ \\
\hline & \multicolumn{5}{|l|}{$\mathrm{cm}$} \\
\hline Total coronary flow & 80 & $16.9 \pm 0.5$ & $18.3 \pm 0.4$ & $10.7 \pm 1.0 \S$ & $12.9 \pm 0.3 \mp \$ \pi$ \\
\hline (ml/g per $\mathrm{min})$ & 140 & $22.0 \pm 0.5$ & $24.3 \pm 0.5 \ddagger$ & $16.7 \pm 1.0 \S$ & $19.0 \pm 0.4 \ddagger^{\prime \prime} \pi$ \\
\hline Left ventricular flow & 80 & $18.5 \pm 0.7$ & $19.9 \pm 0.4$ & $11.0 \pm 1.0 \S$ & $13.5 \pm 0.2 \ddagger \S \pi$ \\
\hline (ml/g per min) & 140 & $23.7 \pm 0.8$ & $26.3 \pm 0.5 \ddagger$ & $17.6 \pm 1.0 \S$ & $20.1 \pm 0.3 \ddagger^{\prime \prime \pi}$ \\
\hline Right ventricular flow & 80 & $10.7 \pm 0.5$ & $12.0 \pm 0.4$ & $9.1 \pm 1.0$ & $9.8 \pm 0.7$ \\
\hline \multirow[t]{2}{*}{ (ml/g per $\mathrm{min})$} & 140 & $15.1 \pm 0.7$ & $16.5 \pm 1.6$ & $12.3 \pm 1.4$ & $13.6 \pm 1.2$ \\
\hline & 80 & $1.06 \pm 0.02$ & $1.11 \pm 0.04$ & $0.87 \pm 0.02 \S$ & $0.82 \pm 0.04 \S$ T \\
\hline Inner/outer & 140 & $1.24 \pm 0.05$ & $1.25 \pm 0.04$ & $1.15 \pm 0.06$ & $1.10 \pm 0.06$ \\
\hline
\end{tabular}

Results are mean $\pm \mathrm{SE}$ of the number of hearts shown in parentheses. Flow values are expressed per gram wet tissue. Other abbreviations and statistical notations are the same as in Tables I and II. 
cardiac hypertrophy due to renal hypertension in rats is associated with a depressed coronary reserve $(7,17)$ and diminished contractile performance of the left ventricle (4-7). These results contrast with our previous findings of improved contractile performance in hypertrophied hearts from female rats trained by a chronic swimming program (12). In the latter study coronary flow per gram of tissue was normal in the hypertrophied hearts.

In the present investigation we examined whether the additional hypertrophic stimulus of physical training, and its attendant salutary cardiac effects, may be manifested in the hypertensive heart, or whether the additional burden of training, by exaggerating the hypertrophy, may indeed be harmful. Our results indicate that virtually all of the cardiac adaptations due to training may be superimposed on the hypertensive heart. This phenomenon was initially apparent by the relative increase in heart mass in the experimental groups. In the swimming group (Sw), the increase in heart mass was $30 \%$, while in the hypertensive group (H) it was $46 \%$. Accordingly, the increase in heart mass was $76 \%$ in the swimming hypertensive group (H-Sw). The manner in which the heart enlarged also showed a similar pattern. In Sw, chamber enlargement was evidenced by an increase in end-diastolic volume compared with controls (C). This increase was similar to that for heart mass, so that the end-diastolic volume normalized per gram tissue was similar to control. Conversely, end-diastolic volume was the same in $\mathbf{H}$ and $\mathbf{C}$, and end-diastolic volume normalized per gram was lower in $\mathrm{H}$. Although normalized end-diastolic volume was lower in $\mathrm{H}-\mathrm{Sw}$ than in $\mathrm{C}$ and the same as in $\mathrm{H}$, the absolute enddiastolic volume was greater in $\mathrm{H}-\mathrm{Sw}$ than in either $\mathrm{H}$ or $\mathrm{C}$. Thus, the findings for end-diastolic volume in $\mathrm{H}-\mathrm{Sw}$ suggest that an eccentric hypertrophy was superimposed upon a concentric hypertrophy.

The findings of additional hypertrophy in $\mathrm{H}-\mathrm{Sw}$ are consistent with the findings of Pfeffer et al. (18) in swim-trained spontaneously hypertensive female rats. These authors observed a larger left ventricular volume and stroke volume in trained spontaneously hypertensive rats but no significant differences in cardiac output even though heart rate was not significantly different. In the present study absolute cardiac output (milliliters per minute) at the same heart rate was $37 \%$ greater in $\mathrm{H}-\mathrm{Sw}$ than $\mathrm{H}$ at the high preload at $140 \mathrm{~cm}$ aortic height. End-diastolic volume (milliliter) was $17 \%$ greater in $\mathrm{H}-\mathrm{Sw}$, suggesting that chamber enlargement partially accounted for the greater absolute cardiac output in H-Sw. However, when assessing intrinsic pump performance by normalizing results for LV dry weight, enddiastolic volume per gram was not different, but cardiac output per gram was significantly greater in $\mathrm{H}-\mathrm{Sw}$, suggesting that improvement in muscle function had occurred in $\mathrm{H}-\mathrm{Sw}$. This was indeed the case as indicated by greater values for ejection fraction, fractional shortening, and mean Vcf in this group. Thus, the greater pumping ability in $\mathrm{H}-\mathrm{Sw}$ was accomplished by chamber enlargment in proportion to the increase in muscle mass and greater muscle shortening. In addition, normalized results for pump and muscle function in $\mathrm{H}-\mathrm{Sw}$ were similar to or greater than those in $\mathrm{C}$, suggesting that training by swimming can reverse the deleterious functional effects of hypertension.
The possibility must be considered that the depressed cardiac function in the hypertensive hearts resulted from the diminution in coronary flow, possibly causing myocardial ischemia. In the aqueous perfusion medium, oxygen content is very low, necessitating a high coronary flow for adequate oxygenation. Thus, it is possible that the lower coronary reserve capacity in $\mathbf{H}$ prevented adequate oxygenation and may have been responsible for the lesser performance in this group. An increased coronary resistance and a reduction in coronary flow reserve have been demonstrated in hypertensive rats, dogs, and humans $(7,17$, 19-21), although in vivo absolute coronary flow at rest is usually normalized or may be increased because of the increased aortic pressure $(21,22)$. Coronary flow in hypertensive hearts in the first series of experiments was reduced by $35 \%$ compared with controls when hearts were perfused under the highest loading condition, and oxygen consumption was similarly diminished (Table II). Although coronary resistance was not calculated, since ADP was the same in all comparisons, resistance must have varied inversely with flow. Also, myocardial extraction of oxygen from the perfusate was slightly but significantly higher in hypertensive than in control hearts. These findings might suggest the presence of myocardial ischemia, except that effluent $L / P$ ratios were not significantly different from $L / P$ ratios in control hearts. Furthermore, metabolic parameters related to possible ischemia in the hearts in which mechanics were measured were similar or more "abnormal" in $\mathrm{H}-\mathrm{Sw}$ than in $\mathrm{H}$. That is, myocardial oxygen extraction and the effluent $\mathrm{L} / \mathrm{P}$ ratio were the same or higher in $\mathrm{H}-\mathrm{Sw}$ than in $\mathrm{H}$. In the second series in which microspheres were used, calculated subendocardial flow and oxygen delivery were similar in $\mathrm{H}-\mathrm{Sw}$ and $\mathrm{H}$. However, hearts of $\mathrm{H}-\mathrm{Sw}$ had higher values than hearts of $\mathrm{H}$ for stroke work, ejection fraction, fractional shortening, and Vcf. In fact many of these values in $\mathrm{H}-\mathrm{Sw}$ were greater or equal to those found in control hearts. If ischemia was a factor, the same or a greater effect should have been seen in hearts of hypertensive swimmers.

The detailed studies of coronary flow and myocardial flow distribution using microspheres (Table III) also fail to support evidence of myocardial ischemia at high ADP. There were significant decreases in the inner to outer wall flow ratios in hearts of hypertensive animals and hypertensive swimmers with the aortic chamber height set at $80 \mathrm{~cm} \mathrm{H}_{2} \mathrm{O}$ but this ratio was normal at $140 \mathrm{~cm} \mathrm{H}_{2} \mathrm{O}$ of aortic height, a condition under which cardiac function was depressed in the hearts of the hypertensive animals and where it had been normalized in the hearts of the hypertensive swimmers. In view of the possible ischemia when hearts of $\mathrm{H}$ and $\mathrm{H}-\mathrm{Sw}$ were initially perfused at $80 \mathrm{~cm}$ aortic pressure, the possibility must be considered that during subsequent perfusions at higher aortic pressures the ischemic damage would persist and affect final performance. This too should have resulted in equal depression of $\mathrm{H}$ and $\mathrm{H}-\mathrm{Sw}$.

That the depression of muscle function in $\mathrm{H}$ is separate from the coronary vascular defect is supported by the observation of depressed velocity of contraction in papillary muscles from hypertensive rats in experiments that do not rely on coronary perfusion (4). The fact that improvement of myocardial function 
in $\mathrm{H}-\mathrm{Sw}$ occurred in series number one in which restoration of the coronary flow responses were not seen also indicates a direct myocardial basis for the alterations. Fig. 4 shows the relationship between actin activated myosin ATPase values reported previously (10) and measurements of Vcf obtained at $140 \mathrm{~cm}$ aortic height in the present experiments. There is almost perfect linearity between the enzymatic activity and mechanical values. Such linearity would be extremely unlikely if oxygen deprivation alone was responsible for the depressed cardiac function in $\mathrm{H}$.

There was no improvement in coronary flow in hearts of hypertensive swimmers compared with hypertensive sedentary hearts in series number one but significant differences were found in series number two. The reasons for this variability are not apparent but the finding suggests that in some circumstances partial reversal of the vascular defect may be observed in hypertensive hearts. It would be important to attempt to clarify this issue in future studies. Several reports indicate that vascular growth does not keep pace with myocardial growth in hypertrophy of systolic overload (23-26). It has recently been observed that myocardial flow reserve and microvascularity, which diminish during the developmental phases of hypertension in spontaneous hypertensive rats, recover as the hypertrophy progresses (26), suggesting that the vascular deficits in hypertension are not fixed phenomena. On the other hand, physical training has been reported to result in increased capillary density in rats $(27,28)$. Since heart mass was greater in $\mathrm{H}-\mathrm{Sw}$ than in $\mathrm{H}$, but coronary flow per gram tissue was the same, or even greater in $\mathrm{H}-\mathrm{Sw}$, it is likely that active vascular growth occurred in the hypertensive swimmers. Thus, the changes in flow observed in our study in hypertensive swimmers may be a result of the
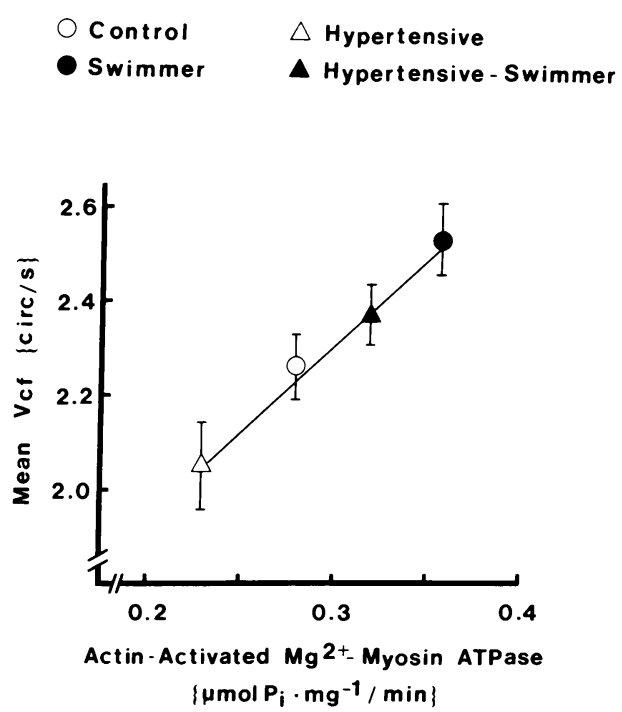

Figure 4. The relationship between mean Vcf and actin-activated $\mathrm{Mg}^{2+}$-myosin ATPase. Mean Vcf was obtained at the high preload condition at an aortic height of $140 \mathrm{~cm}$. Values for ATPase have been reported previously (10). $\bigcirc$, control; ๑, swimmer; $\Delta$, hypertensive; $\triangle$, hypertensive-swimmer. balancing of the effects of hypertension with those of physical training on myocardial perfusion.

The data on coronary flow distribution are of interest in that most studies in the literature have been performed with pressure of $\sim 80 \mathrm{~cm}$ of $\mathrm{H}_{2} \mathrm{O}$. The present results indicate that at high preloads, subendocardial underperfusion could exist in hypertrophied hearts from hypertensive animals. Thus, in hearts where coronary reserve is compromised, heart perfusions should be conducted at higher perfusion pressures. This is also suggested by the metabolic measurements. In each group of hearts oxygen extraction decreased with increasing aortic pressure, and in the hearts from hypertensive groups, lactate production and effluent $\mathrm{L} / \mathrm{P}$ ratios also declined with increasing aortic pressures. This was true despite the finding that as afterload was raised, myocardial energy requirement as measured by oxygen consumption also increased.

It is likely that the mechanism of the depressed contractile and pump function in the hearts of the hypertensive animals in this study and the reversion back towards normal in hypertensive swimmers is a result of basic biochemical changes in the myocardium. This is supported by our recent report on contractile proteins in these models demonstrating that actomyosin, myosin, and actin activated myosin ATPase activities from the myocardium of hypertensive rats are depressed, but that in hearts of hypertensive swimmers, the contractile protein abnormalities were reversed (10). These changes in ATPase activity are paralleled by alterations in the balance of cardiac myosin isoenzymes. Similarly, biochemical control of cardiac contractile function is probably exerted at the level of the sarcolemma and of the sarcoplasmic reticulum but these systems have not been explored systematically in the current model of hypertension plus physical training.

The present experiments provide further evidence that the hypertrophy caused by systolic overload, which in some instances leads to depressed myocardial function, and that caused by repeated swimming are markedly different. If hypertrophy was a continuum, the superimposition of a swimming load upon hearts of hypertensive animals in this study might have been expected to exaggerate the abnormalities observed in the hypertensive hearts. The hypertrophy associated with pure swimming clearly causes function, biochemistry, and capillarity to shift in the opposite direction to that associated with systolic overload. The fact that adding swimming to hypertension causes a reversal of the cardiac effects of hypertension alone, indicates the mechanisms responsible for these two hypertrophic processes are separate and distinct.

The results of these studies are provocative both in terms of fundamental mechanisms of cardiac adaptation and in terms of potential application to humans. Clearly, if heart function and biochemical changes secondary to disease conditions such as aortic stenosis or hypertension, where there is pathologic cardiac hypertrophy, can be reversed or improved by physical training, physical training might have a therapeutic role in the treatment of patients with these disorders. It is important to note in the present investigation that beneficial cardiac effects of training occurred in hearts of hypertensive animals even 
though there was no effect of training on blood pressure. However, the possibility must be considered that prolonged physical training would finally result in a worsening of heart function in the already pathologically hypertrophied heart. Also, it must be emphasized that the current experiments are of relatively short duration in very specific animal models of hypertrophy, the renal hypertensive rat and the swimming female rat. Physical training by running and by swimming in rats has somewhat different cardiac effects (11) and these responses differ somewhat in male and in female rats $(12,29)$, and in fact not all models of physical training demonstrate enhanced myocardial contractility (8). Therefore, many more fundamental questions must be explored before this concept can be applied to clinical states.

\section{Acknowledgments}

We wish to thank Alwyn Murphy for his excellent technical assistance and Dr. Abraham Alfaro for his preliminary work on this subject. We also wish to thank Ms. Janet Holwell and Mrs. Lori Fields for their expert secretarial assistance, and Ms. Claudette Davis for her editorial assistance.

This work was supported in part by National Institutes of Health grant HL-15498 and the Roger and Barbara Michaels Family Fund, Inc.

\section{References}

1. Wikman-Coffelt, J., W. W. Parmley, and D. T. Mason. 1979. The cardiac hypertrophy process. Analyses of factors determining pathological vs. physiological development. Circ. Res. 45:697-707.

2. Williams, J. F., Jr., and R. D. Potter. 1974. Normal contractile state of hypertrophied myocardium after pulmonary artery constriction in the cat. J. Clin. Invest. 54:1266-1271.

3. Broughton, A., and P. I. Korner. 1983. Basal and maximal inotropic state in renal hypertensive dogs with cardiac hypertrophy. Am. J. Physiol. 245:H33-H41.

4. Capasso, J. M., J. E. Strobeck, A. Malhotra, R. Aronson, J. Scheuer, and E. H. Sonnenblick. 1982. Contractile behavior of rat myocardium after reversal of hypertensive-hypertrophy. Am. J. Physiol. H882-H889.

5. Jacob, R., G. Ebrecht, I. Kammerait, I. Medugorac, and M. WendtGallitelli. 1977. Myocardial function in different models of cardiac hypertrophy. An attempt at correlating mechanical, biochemical, and morphological parameters. Basic Res. Cardiol. 72:160-167.

6. Averill, D., C. Ferrario, R. Tarezi, S. Sen, and R. Barbus. 1976. Cardiac performance in rats with renal hypertension. Circ. Res. 38:280 288.

7. Alfaro, A., T. Schaible, A. Malhotra, T. Yipintsoi, and J. Scheuer. 1983. Impaired coronary flow and ventricular function in hearts of hypertensive rats. Cardiovasc. Res. 17:553-561.

8. Scheuer, J., and C. M. Tipton. 1977. Cardiovascular adaptations to physical training. Ann. Rev. Physiol. 39:221-251.

9. Scheuer, J., and A. K. Bhan. 1979. Cardiac contractile proteins: ATPase activity and physiologic function (brief review). Circ. Res. 45:112.

10. Scheuer, J., A. Malhotra, C. Hirsch, J. Capasso, and T. F. Schaible. 1982. Physiologic cardiac hypertrophy corrects contractile protein ab- normalities associated with pathologic hypertrophy in rats. J. Clin. Invest. 70:1300-1305.

11. Schaible, T. F., and J. Scheuer. 1979. Effects of physical training by running or swimming on ventricular performance of rat hearts. $J$. Appl. Physiol. 46:854-860.

12. Schaible, T. F., and J. Scheuer. 1981. Cardiac function in hypertrophied hearts from chronically exercised female rats. J. Appl. Physiol. 50:1140-1145.

13. Bersohn, M. M., and J. Scheuer. 1977. Effects of physical training on end-diastolic volume and myocardial performance of isolated rat hearts. Circ. Res. 40:510-516.

14. Hohorst, H. 1965. L-(+)-Lactate: determination with lactic dehydrogenase and DPN. In Methods of Enzymatic Analysis. H. U. Bergmeyer, editor. Academic Press, Inc., New York. 266-270.

15. Segal, S., A. Blair, and J. Whydaarden. 1956. An enzymatic spectrophotometric method for the determination of pyruvic acid in blood. J. Lab. Clin. Med. 137-143.

16. Zar, J. H. 1974. Biostatistical Analysis. Prentice-Hall, Englewood Cliffs, NJ. 105-107.

17. Wangler, R. D., K. G. Peters, M. L. Marcus, and R. J. Tomanek 1982. Effects of duration and severity of arterial hypertension and cardiac hypertrophy on coronary vasodilator reserve. Circ. Res. 51:10-18.

18. Pfeffer, M. A., B. A. Ferrell, J. M. Pfeffer, A. K. Weis, M. C. Fishbein, and E. D. Froelich. 1978. Ventricular morphology and pumping ability of exercised spontaneously hypertensive rats. Am. J. Physiol. 235:H193-H199.

19. Mueller, T. M., M. L. Marcus, R. E. Herber, J. A. Young, R. W. Barnes, and F. M. Abboud. 1978. Effect of renal hypertension and left ventricular hypertrophy on the coronary circulation in dogs. Circ. Res. 42:543-549.

20. Marcus, M. L., T. M. Mueller, J. A. Gascho, and R. E. Kerber. 1979. Effects of cardiac hypertrophy secondary to hypertension on the coronary circulation. Am. J. Cardiol. 44:1023-1028.

21. Strauer, B. E. 1980. Hypertensive Heart Disease. Springer-Verlag, Berlin.

22. Wicker, P., R. C. Tarazi, and K. Kobayashi. 1983. Coronary blood flow during the development and regression of left ventricular hypertrophy in renovascular hypertensive rats. Am. J. Cardiol. 51:17441749.

23. Henquell, L., C. L. Odorogg, and C. Honig. 1977. Intercapillary distance and capillary reserve in hypertrophied rat hearts beating in situ. Circ. Res. 41:400-407.

24. Ljingqvist, A., and G. Unge. 1973. The proliferative activity of the myocardial tissue in various forms of experimental cardiac hypertrophy. Acta Path. Microbiol. Scand. A Pathol. 81:233-240.

25. Lund, D. D., and R. J. Tomanek. 1978. Myocardial morphology in spontaneously hypertensive and aortic-constricted rats. Am. J. Anat. 152:141-152.

26. Tomanek, R. J., J. C. Searls, and P. A. Lachenbruch. 1982. Quantitative changes in the capillary bed during developing, peak, and stabilized cardiac hypertrophy in the spontaneously hypertensive rat. Circ. Res. 51:295-304.

27. Bloor, C. M., and A. S. Leon. 1970. Interaction of age and exercise on the heart and its blood supply. Lab. Invest. 22:160-165.

28. Tomanek, R. J. 1969. Effects of age and exercise on the extent of the myocardial capillary bed. Anat. Rec. 167:55-62.

29. Schaible, T. F., S. Penpargkul, and J. Scheuer. 1981. Cardiac responses to exercise training in male and female rats. J. Appl. Physiol. 50:112-117. 